\title{
Shear-Thinning Rheology of the Abuse-Deterrent Dosage Form Extracts
}

David J Mastropietro, Srinath Muppalaneni, Arghavan Kariman and Hossein Omidian*

Department of Pharmaceutical Sciences, College of Pharmacy, Nova Southeastern University, Fort Lauderdale, Florida, USA

\begin{abstract}
Prescription drug abuse has become a worldwide public health issue. Of particular concern is the dose-dumping effect caused by co-ingestion of ethanol which leads to premature rapid release of the drug load. Also, medications can be crushed and dissolved in ethanol for extraction to allow for parenteral routes of administration. To combat this abuse, the pharmaceutical industry has begun to develop dosage forms that are abuse deterrent. In this study, we investigated the use of a cone-and-plate rheometer to evaluate the rheological properties of polyethylene oxide (a common excipient in abuse deterrent dosage forms) in water-alcohol mixtures. This polymer prevents rapid drug dissolution in alcohol by forming a viscous gel in the presence of solvents to discourage intravenous abuse. Our results showed that $2 \% \mathrm{w} / \mathrm{v}$ polyethylene oxide in hydro-alcoholic solvents behaved non-Newtonian. Rheograms showed a non-linear profile where apparent viscosity was decreased as a function of increasing shear rate, and was fit to a Herschel-Bulkley fluid model. The greater viscosities seen in test solutions at low shear rates may prevent rapid ethanol dissolution of drug, and provide difficulty when dissolving a product in hydro-alcoholic solutions. However, high shear rates such as that seen in rapid mixing or in a needle and syringe are likely to adversely affect the deterrence capacity of polyethylene oxide in solutions.
\end{abstract}

Keywords: Abuse deterrent; Tamper resistant; Rheology; Dose dumping; Ethanol; Shear-thinning

\section{Introduction}

With the widespread prevalence of prescription drug abuse, the introduction of abuse-deterrent and tamper-resistant medications into the marketplace has never been more important. Based on the low number of approved products utilizing these novel technologies, producing formulations that show resistance to common methods of abuse is challenging. One of the biggest challenges is to manufacture a dosage form that is safe and effective when taken as directed, yet can still prevent or deter its misuse and abuse.

Solid oral dosage forms are the most commonly prescribed and also abused formulations in the public hands. One common method of their abuse is the extraction of the active drug from the dosage form using aqueous or hydro-alcoholic solutions in an attempt to make a concentrated solution of the drug for parenteral use [1,2]. Additionally, co-ingesting certain medications with ethanol can lead to accelerated dissolution and rapid absorption of the drug into the blood stream to produce euphoric effects. This is particularly important for long-acting and extended release dosage forms where rapid release of a large drug load can be dangerous and even fatal [3]. The nature of the excipients as well as the exposure time to the ethanol makes the behavior of a dosage form to hydro-alcoholic solutions very unpredictable [4]. This has forced formulation scientists to rethink and evaluate the ethanol sensitivity of both active and inactive components utilized in dosage forms containing abusable drugs. This research can ultimately determine excipients and formulations that are more and most insensitive to this type of misuse and abuse. An ideal formulation to prevent abuse would be one that can impede drug extraction in hydro-alcoholic solutions, even if the dosage form is chewed, crushed, or physically tampered with in an attempt to reduce particle size or destroy any long acting mechanism. Additionally, a formulation can also be made to change its physical properties upon being mixed with ethanol or aqueous solutions to form a mixture that is incapable of being drawn into a syringe or passed through a filter before being injected. This may be accomplished with the use of gelling or viscosity modifying agents that trap the drug or increase the internal friction of the extract to an extent that it prevents syringeability and filtration. Various hydrophilic and ethanol soluble polymers can be used for this purpose in formulations to prevent parenteral abuse [5].

One example polymer suited for this application is high molecular weight polyethylene oxide. This non-ionic and fast hydrating polymer is commonly used in many solid oral dosage forms including direct compression tablets, controlled release matrix systems and hot melt extrusion products [6]. This polymer is also listed as an ingredient in all currently marketed products with an abuse deterrence potential (i.e., Exalgo, Nucynta ER, Oxycontin', Oxecta, and Opana ER'). However, it is not well established how this polymer behaves in various alcohol concentrations under shear. Therefore, the objective of this study was to investigate the rheological properties of Polyethylene Oxide (PEO) when in the presence of varying hydro-alcoholic solutions using a Wells-Brookfield cone-and-plate rheometer.

\section{Materials and Methods}

\section{Materials}

Polyethylene Oxide (PEO) water-soluble resin (Sentry ${ }^{\mathrm{TM}}$ Polyox ${ }^{\mathrm{Tm}}$ WSR Coagulant NF) was supplied by Dow Chemical (Midland, MI), ethyl alcohol 200 Proof USP grade was obtained by Pharmco Products Inc (Brookfield, CT). Millipore filtered water $(\approx 16 \mathrm{M} \Omega \bullet \mathrm{cm})$ was used to prepare all hydro-alcoholic solutions.

\section{Solution Preparation}

Hydro-alcoholic solutions were prepared using 200 proof ethyl

*Corresponding author: Hossein Omidian, Associate Professor, Department of Pharmaceutical Sciences, College of Pharmacy, Nova Southeastern University, 3200 South University Drive, Fort Lauderdale, FL, 33314, USA; Tel: 1-954-262-1334; E-mail: omidian@nova.edu

Received July 29, 2013; Accepted December 20, 2013; Published December 27, 2013

Citation: Mastropietro DJ, Muppalaneni S, Kariman A, Omidian H (2013) ShearThinning Rheology of the Abuse-Deterrent Dosage Form Extracts. J Develop Drugs 2: 114. doi:10.4172/2329-6631.1000114

Copyright: ( 2013 Mastropietro DJ, et al. This is an open-access article distributed under the terms of the Creative Commons Attribution License, which permits unrestricted use, distribution, and reproduction in any medium, provided the origina author and source are credited. 
alcohol as 0, 5, 20, 40, 60, 80, 100\% v/v alcohol concentration. These solvents were used to make $2 \% \mathrm{w} / \mathrm{v}$ solutions of PEO. The PEO was first passed through a $250 \mu \mathrm{m}$ mesh screen, and then the powder directly dispersed into the solvents. Solutions were then periodically agitated during the hydration stage, and further stored for a minimum of 24 hours at room temperature prior to use.

\section{Rheological Measurements}

Continuous shear rheometry was performed using a WellsBrookfield cone-and-plate rheometer (DV-III Ultra, Brookfield Engineering, USA) (Figure 1) having a standard cup embedded with a temperature probe and circulating water bath. Measurements were taken with an attached cone of radius $1.2 \mathrm{~cm}$, cone angle of $3^{\circ}$, and at a controlled temperature of $24.96 \pm 0.3^{\circ} \mathrm{C}$. Test solutions were first centrifuged at $1500 \mathrm{rpm}$ for $5 \mathrm{~min}$ to remove entrapped air bubbles, and then a $0.5 \mathrm{ml}$ sample was carefully applied to the middle of the plate and allowed to equilibrate for 2 minutes. Samples where then subjected to increasing shear rates ranging from 2 to $50 \mathrm{sec}^{-1}$. After 15 seconds of reaching each rate $\left(2,10,20,30,40,50 \mathrm{sec}^{-1}\right)$, a reading was taken to generate the individual rheograms. Due to the suspension nature of the PEO in $100 \%$ ethanol, this sample was shaken prior to dispensing onto the plate to evenly disperse the undissolved particles.

\section{Results and Discussion}

\section{Solution preparation}

The high molecular weight PEO used in this study formed a clear to slightly cloudy solution in water and within all the hydro-alcoholic



Figure 1: Wells-Brookfield cone-and-plate rheometer

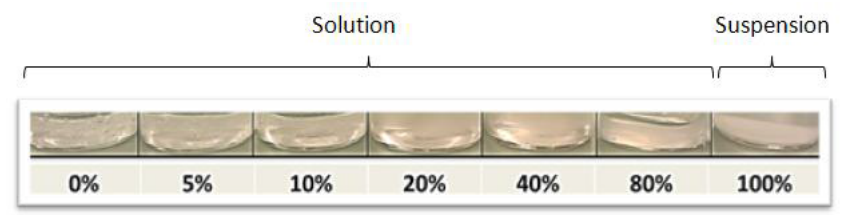

Figure 2: Solubility of $2 \mathrm{wt} \%$ PEO in hydro-alcoholic solutions.



Figure 3: Viscosity of $2 \mathrm{w} / \mathrm{v} \%$ PEO in hydro-alcoholic solutions at a shear rate of 2,20 , and $40 \mathrm{sec}^{-1}$.



Figure 4: Viscosity of $2 \mathrm{w} / \mathrm{v} \%$ polyethylene oxide solutions at different ethanol $(\mathrm{EtOH})$ concentrations and shear rates.

concentrations. However, a visible particulate suspension was formed when PEO was mixed in a $100 \%$ ethanol as seen in Figure 2.

\section{Rheological measurements}

PEO displayed different viscosities when mixed with hydroalcoholic solutions in which it was soluble. Shown in Figure 3, at low shear rate of $2 \mathrm{sec}^{-1}$, the solution viscosity was gradually increased with increase in alcohol concentration, reached its maximum at $60-80 \%$ ethanol concentration, and then dropped sharply afterwards where PEO was practically insoluble in extremely rich alcoholic solution and pure alcohol. A similar trend was observed at two other shear rates of 20 and $40 \mathrm{sec}^{-1}$. At $20 \mathrm{sec}^{-1}$, in all solutions the viscosities were decreased to near $20 \%$ of their corresponding values at shear rate of $2 \mathrm{sec}^{-1}$, and even lower values were observed when shear rate was increased to $40 \mathrm{sec}^{-1}$.

To better understand the effect of shear rate when PEO dissolved in different hydro-alcoholic solutions, the viscosity values were plotted over the whole spectrum of applicable shear rates of $0-50 \mathrm{sec}^{-1}$ as shown in Figure 4. A rapid change in viscosity was seen as shear rates were increased, and the solutions apparently displayed a shear thinning effect. A rapid decline in each solution's resistance to applied force occurred almost immediately as the shear rate increased from 2 to 10 $\mathrm{sec}^{-1}$ and the viscosity became almost three to four times as less.

Rheocalc software (V3.3, Brookfield Engineering) was used to determine the best fit mathematical model for the viscoelastic solution behaviors. The non-Newtonian solutions were analyzed and best fit to the Herschel-Bulkley equation as shown in Figure 5. This equation can be shown as follows:

$$
\tau=\tau_{0}+K(\gamma)^{n}
$$






Figure 5: Experimentally derived raw data and Herschel-Bulkley model fitted curve rheograms for various hydro-alcoholic solutions of $2 \%$ polyethylene oxide.

\begin{tabular}{|c|c|c|c|c|}
\hline EtOH (\%) & $\mathbf{K}(\mathbf{c P})$ & $\mathbf{n}$ & $\tau_{\mathrm{o}}$ & Confidence of Fit (\%) \\
\hline 0 & 7753 & 0.44 & 53.5 & 99.8 \\
\hline 20 & 5865 & 0.53 & 89.2 & 99.7 \\
\hline 40 & 6642 & 0.53 & 93.1 & 99.8 \\
\hline 80 & 6800 & 0.53 & 103.4 & 99.4 \\
\hline
\end{tabular}

Table 1: Experimental parameters for Herschel-Bulkley model.

Where $\tau$, shear stress; $\tau_{0}$, yield stress; $\mathrm{K}$, consistency index; $\gamma$, shear rate; and $\mathrm{n}$, flow index.

The rheograms profiles of each solution show a non-linear trend where apparent viscosity (slope) is decreased as a function of increasing shear rate. The flow index $(\mathrm{n})$ values $<1$ confirms the shear thinning effect observed as detailed in Table 1.

Knowing the fact that non-Newtonian fluids such as hydroalcoholic solutions of PEO show different rates of flow as a function of the pressure being applied and the geometry they are forced to flow in, the deterrence capacity of the deterrent excipient in such pharmaceutical dosage form can critically be a function of how fast the extract will be drawn into the syringe and the syringe needle gauge [7].

\section{Conclusion}

Using a cone and plate rheometer, it was shown that both aqueous and highly concentrated hydro-alcoholic solutions of 2\% PEO displayed high viscosity only at very low shear rates. This high viscosity may prevent rapid release of a drug in the presence of alcoholic drinks by slowing dissolution and diffusion of the drug out of a dosage form. For parenteral abuse, this would also appear to make an extract hard to drawn into a syringe and inject. However, our results also show that if the solutions are agitated or mixed, there would be a substantial decrease in solution viscosity that may possibly negate the deterrence capacity of the PEO. The concentration of ethanol used in this study covers the full range that can be found both in alcoholic drinks causing "dose dumping". The extremely high shear rates that are seen when a fluid is drawn up into a syringe should make these types of solutions easy to pass through a needle and be injected with ease. However, the appearance of a thick solution at low shear rate may be enough to deter an abuser from using such extracts for parenteral injection. Therefore, the effectiveness of PEO as a gel forming excipient in abuse-deterrent formulations appears to be critically dependent on the ethanol concentration and the level of mixing (shear rate) used.

\section{Acknowledgement}

Funding: Support for this project was generously provided by Nova Southeastern University's President's grant \#335867 and Health Profession Division Research Grant \#335829.

\section{References}

1. Betancourt AO, Gosselin PM, Vinson RK (2013) New immediate release formulation for deterring abuse of methadone. Pharm Dev Technol 18: 535-543.

2. McLean S, Bruno R, Brandon S, de Graaff B (2009) Effect of filtration on morphine and particle content of injections prepared from slow-release oral morphine tablets. Harm Reduct J 6: 37.

3. Johnson FK, Ciric S, Boudriau S, Kisicki J, Stauffer J (2012) Effects of alcoho on the pharmacokinetics of morphine sulfate and naltrexone hydrochloride extended release capsules. J Clin Pharmacol 52: 747-756.

4. Fadda HM, Mohamed MA, Basit AW (2008) Impairment of the in vitro drug release behaviour of oral modified release preparations in the presence of alcohol. Int J Pharm 360: 171-176.

5. Mastropietro D, Muppalaneni S, Omidian H (2013) Abuse-deterrent Behavior of Polyethylene Oxide to Hydroalcoholic Solutions [Poster]. PharmForum'13 hosted by University of Arkansas and AAPS southern regional discussion group, Little Rock, AK, 2013.

6. (2013) Polyox ${ }^{\mathrm{TM}}$ water-soluble resins. We can help create better medicine. Dow Wolff Cellulosics.

7. Allahham A, Mainwaring D, Stewart P, Marriott J (2004) Development and application of a micro-capillary rheometer for in-vitro evaluation of parenteral injectability. J Pharm Pharmacol 56: 709-716. 